\title{
Equilibration and Order in Quantum Floquet Matter
}

\author{
R. Moessner ${ }^{1}$ and S. L. Sondhi ${ }^{2}$ \\ ${ }^{1}$ Max-Planck-Institut für Physik komplexer Systeme, 01187 Dresden, Germany \\ ${ }^{2}$ Department of Physics, Princeton University, Princeton, New Jersey 08544, USA
}

\begin{abstract}
Equilibrium theormodynamics is characterized by two fundamental ideas: thermalisation-that systems approach a late time thermal state; and phase structure-that thermal states exhibit singular changes as various parameters characterizing the system are changed. We summarise recent progress that has established generalizations of these ideas to periodically driven, or Floquet, closed quantum systems. This has resulted in the discovery of entirely new phases which exist only out of equilibrium, such as the $\pi$-spin glass or Floquet time crystal.
\end{abstract}

Introduction: Remarkable progress in the physics of closed quantum systems away from equilibrium has occurred over the last decade. This has been experimental - most strikingly in cold atomic systems, ${ }^{1}$ computational - often involving quantum information ideas, ${ }^{2}$ and intellectual - ranging from a systematic use of entanglement ideas to the long sought demonstration that localization exists in many body systems. ${ }^{3}$ Here, we report very recent progress building particularly on the latter, in our understanding of periodically driven or Floquet many body systems.

Closed Floquet systems comprise a vast family of systems generally defined by 'drives' or time dependent Hamiltonians with $\mathcal{H}(t+T)=\mathcal{H}(t)$ for a fixed period $T$. The promise of Floquet systems is that the periodic drive can lead to new physical phenomena, but their peril is the risk of heating up to a "fully scrambled" or "infinite temperature" state, supporting no non-trivial correlations as all configurations occur with the same probability.

The progress reviewed here has established that the peril can be avoided; that interesting long time steady states can be obtained; and that sharply different behaviors can be distinguished and classified, providing generalizations of the foundational thermodynamic notions of thermalization and phase structure ${ }^{42}$ into the nonequilibrium regime. Indeed, Floquet systems arguably represent the maximum known extension of equilibrium phase structure in that generic driven systems lacking periodicity are believed to heat to infinite temperature. Pioneering experiments ${ }^{4-6}$ have very recently started exploring this universe of many body Floquet drives.

Our viewpoint is statistical mechanical and restricted to closed/isolated systems. There is also a large and older literature on single particle Floquet systems ${ }^{7}$ and much recent work on using Floquet physics to engineer nontrivial Hamiltonians as well as on open system physics to use such engineering to interesting ends. We make contact with this larger Floquet universe only where it intersects with our main theme and direct the reader to the literature for this complementary work ${ }^{8-17}$.

Floquet basics: Most broadly, the quantum mechanics of closed systems is concerned with their unitary time evolution governed by the Schrödinger equation $(\hbar=1)$

$$
i \frac{d}{d t} U\left(t, t_{0}\right)=\mathcal{H}(t) U\left(t, t_{0}\right)
$$

where $U\left(t, t_{0}\right)$ is the unitary time evolution operator that relates states at time $t_{0}$ to states at time $t$. For completely general $\mathcal{H}(t)$ there is not much else to do than to buckle down and solve (1). For static systems, $\mathcal{H}(t) \equiv \mathcal{H}_{0}$, life is much simpler as $U\left(t, t_{0}\right)=e^{-i\left(t-t_{0}\right) \mathcal{H}_{0}}$, and so we learn vast amounts by solving the eigensystem problem for $\mathcal{H}_{0}$. Specifically, the eigenstates give rise to special, stationary, solutions of the Schrödinger equation that form a basis for general time evolution.

The fundamental difference between the Hamiltonians of Floquet and static systems is that the latter are fully independent of time, while the former are only invariant under discrete time translations by a period $T$. This difference is analogous to the difference between translation invariance of the continuum and of a lattice. There, the former allows us to study the spectrum of the generator of translations (the momentum) while the latter requires that we study the spectrum of the discrete translation operator itself, with states in different bands corresponding to the same quasi-momentum. Correspondingly, for Floquet systems one needs to study the properties of the family of single period time evolution operators

$$
U\left(t_{0}+T, t_{0}\right)=\mathcal{T} e^{-i \int_{t_{0}}^{t_{0}+T} d t^{\prime} \mathcal{H}\left(t^{\prime}\right)}
$$

where $0 \leq t_{0}<T$.

Let us define $U(T)=U(T, 0)$, whose eigenstates

$$
U(T)\left|\phi_{\alpha}\right\rangle=e^{-i \epsilon_{\alpha} T}\left|\phi_{\alpha}\right\rangle
$$

define special solutions of (1), the Floquet eigenstates

$$
\left|\psi_{\alpha}(t)\right\rangle=U(t, 0)\left|\phi_{\alpha}\right\rangle
$$

which satisfy $\left|\psi_{\alpha}(t+T)\right\rangle=e^{-i \epsilon_{\alpha} T}\left|\psi_{\alpha}(t)\right\rangle$. Like the stationary solutions of the static problem, they explicitly exhibit the temporal periodicity of the Hamiltonian and form a basis for general time evolution. The choice of quasienergy $\epsilon_{\alpha}$ is not unique as $\epsilon_{\alpha} \equiv \epsilon_{\alpha}+n_{\alpha}(2 \pi / T)$. This is the freedom in choosing the operator logrithm in $U(T)=e^{-i H_{F} T}$, to obtain what is called the Floquet Hamiltonian $H_{F}$. A final piece of jargon: one refers to a time series spaced $T$ apart as being stroboscopic.

To heat or not to heat: We begin with the textbook thermodynamic viewpoint, which notes that systems without continuous time translation symmetry do not conserve 
energy; in particular in periodically driven systems, energy is conserved only modulo $2 \pi / T$. For generic systems lacking any other local conserved quantities, thermodynamics predicts an entropy maximizing state at late times that is just the infinite temperature state ${ }^{18-20}$, with all local operator expectation values time independent at long times irrespective of the starting state. ${ }^{43}$ We can reach the same conclusion by noting that linear response theory implies absorption at nonzero frequencies and thus a heating cascade that can only terminate at $T=\infty$. In this unique ergodic phase, all Floquet eigenstates must individually yield $T=\infty$ correlations and exhibit volume law enanglement with the maximum thermodynamic entropy. This requirement is an incarnation of the eigenstate thermalisation hypothesis (ETH), originally formulated for static ergodic systems ${ }^{21}$ which states that the value of any local observable in an eigenstate is a smooth function of its energy density, as shown in Fig. 1, so that replacing an exact eigenstate with an ensemble of states around its energy yields the same thermodynamic behaviour. If the system has a finite number of conserved quantities, other than the now missing energy, the late time states can depend on these. An example would be fermion number for a set of interacting fermions. However given the typically macroscopic number of states in each sector defined by these conserved quantities, we expect that each sector exhibits infinite temperature up to global constraints, although it would be interesting to find examples where the sectors exhibit singular changes as the conserved densities are varied.

Existence of the Floquet-ergodic phase and applicability of ETH to its Floquet eigenstates has been confirmed computationally. There is considerable evidence that clean, interacting drives generically give rise to this behavior, as assumed in the following. However, exceptions $^{22}$ and apparent exceptions ${ }^{23,24}$ are known and deserve of more investigation even as there is no good reason to assume that they represent stable behavior.

Leaving such worries aside, the suggestion is that to avoid heating we need $O(N)$ integrals of the of motion, i. e. quantities that commute with $U(T)$, and which can be written as sums of quasi-local terms. There are two known classes of systems where this is the case.

The first class is driven free fermion systems ${ }^{25}$ and equivalent interacting spin systems obtained via JordanWigner transformation in $d=1$. Such systems are described by a quadratic Floquet Hamiltonian

$$
H_{F}=\sum_{\alpha} \epsilon_{\alpha} a_{\alpha}^{\dagger} a_{\alpha}
$$

where for $N$ sites, there are $N$ conserved quantities

$$
I_{\alpha}=a_{\alpha}^{\dagger} a_{\alpha}
$$

For a local $H(t)$, linear combinations of these constants likely always yield quasi-local conserved quantities. We will return to the implications of this below.

The second class is Floquet systems exhibiting many body localization (MBL). Their discovery ${ }^{26-28}$ came as

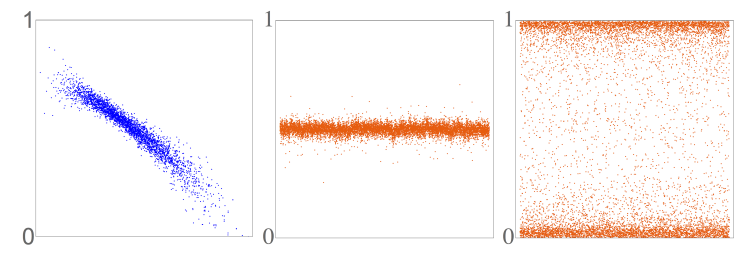

FIG. 1: Eigenstate properties in a local observable in an inhomogeneous system. (left) Undriven system obeying eigenstate thermalisation: the local observalbe is a monotonic function of eigenenergy. (middle) Driven system obeying FloquetETH: observable is constant function of quasienergy. (right) Driven Floquet-MBL system: observable fluctuates strongly even between states with adjacent quasienergies.

a byproduct of the explosion of interest in $\mathrm{MBL}^{3}$, which generalizes the venerable Anderson localization of noninteracting particles to the interacting setting. For these systems, it was established that there exists a set of $O(N)$ spatially localized, mutually commuting, 'l-bit' operators $\tau_{i}^{z}$ (which depend on details of the drive) such that

$$
\left[U(T), \tau_{i}^{z}\right]=0 .
$$

Floquet-MBL is most intuitive when adding a weak drive to a static MBL system (although not restricted to this case). The reference MBL system is itself described by a set of l-bits that commute with its Hamiltonian. The drive flips groups of l-bits only locally, so that the energy difference between initial and final state is bounded above, and it is also nonzero as there generically is no local resonance. Stability of the MBL phase then follows for driving frequencies $2 \pi / T$ high compared to the upper bound, where the system can rather be expected to resemble a set of finite-state Rabi oscillations localized in different regions, which does not heat indefinitely. By contrast, for low driving frequencies at fixed driving amplitudes, absorbing one (or several) quanta of energy $\omega$ gives rise to transitions between the local levels, thereby destroying the MBL phase by local heating. A combination of computational studies, along with more detailed qualitative and analytic arguments, ${ }^{26-29}$ as well as very recent experimental work, ${ }^{4}$ underpin the belief in the existence of this Floquet-MBL to Floquet-ETH transition.

Note that Floquet-MBL systems avoid heating generically — weak perturbations of Floquet-MBL drives that leave the period unchanged are also Floquet-MBL. By contrast, free fermion systems are stable to interactions only when Anderson localized by disorder.

We next discuss how these systems host generalizations of the two central ideas of thermodynamics - of equilibrium and phase structure. We take these in reverse order. Eigenstate Order and Phase structure: As the Floquet$\overline{\mathrm{ETH}}$ phase is the only ergodic phase, all other phases must be localized. To define such phases it is fruitful to generalize the notions of eigenstate order and eigenstate phase transitions from the study of undriven $\mathrm{MBL}^{30}$ to Floquet systems. Eigenstate order exists when individual many body eigenstates exhibit ordering, of which the 


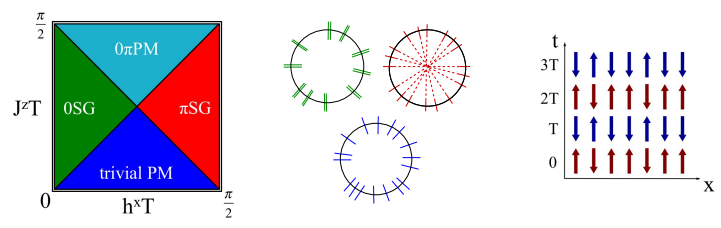

FIG. 2: (left) Phase diagram for the MBL Ising symmetric drives ${ }^{12,31}$ showing $0 \mathrm{SG}$ and $\pi \mathrm{SG}$ phases which are longrange ordered and spontaneously break Ising symmetry, as well as the $0 \pi$-PM and trivial paramagnetic phases without LRO. The $0 \pi \mathrm{PM}$ is an SPT with non-trivial edge modes and can spontaneously break time translation symmetry on its edges. The time crystal $\pi \mathrm{SG}$ is absolutely stable in that its existence is not predicated on the Ising symmetry. (middle): Floquet Eigenstate order: the quasienergy axis running from 0 to $2 \pi / T$ is shown as a circle, with location of Floquet eigenenergies shown, which are distributed randomly $(\mathrm{PM})$, in pairs (0SG) and in pairs diametrically separated by $\pi / T$ ( $\pi \mathrm{SG}$ ). (right): Period doubling in the $\pi \mathrm{SG}$ spatiotemporal order as seen in stroboscopic snapshots.

spectrum exhibits a characteristic signature; at eigenstate phase transitions the eigenstates and eigenvalues can exhibit singular changes as a parameter is varied. ${ }^{44}$ For Floquet systems order can involve non-trivial variations of the eigenstates inside the Floquet period.

To get a sense of how more, and fundamentally new, phases arise ${ }^{31}$ we discuss the simplest setting - that of Floquet-MBL chains with an Ising $\left(\mathbb{Z}_{2}\right)$ symmetry. Consider the binary drive protocol

$$
H(t)=\left\{\begin{array}{l}
-\sum_{s} h_{s} \sigma_{s}^{x}+H_{\mathrm{int}} \quad \text { for } 0 \leq t<T_{1} \\
-\sum_{s} J_{s} \sigma_{s}^{z} \sigma_{s+1}^{z}+H_{\mathrm{int}} \quad \text { for } T_{1} \leq t<T
\end{array}\right.
$$

where $\sigma_{s}^{x}, \sigma_{s}^{z}$ are Pauli-matrix operators at site $s$, and the $h_{s}, J_{s}$ are weakly random about mean values $h$ and $J$ to obtain localization; the additional interaction terms, weaker still to preserve localisation, prevent a possible reduction to free fermions. All terms commute with a global Ising symmetry $P=\prod_{s} \sigma_{s}^{x}$.

This family of drives exhibits exhibits four localized phases. These are shown in the phase diagram Fig 2 for the free fermion limit; with interactions the Floquet ergodic phase will also appear. These phases are characterized as follows in terms of the the spectrum of $U(T)$ and the correlations $\mathcal{C}_{i j}=\left\langle\sigma_{i}^{z} \sigma_{j}^{z}\right\rangle$ at long distance $|i-j| \rightarrow \infty$ of the local Ising odd operators $\sigma_{i}^{z}$, Fig. 2:

- Paramagnet PM (no symmetry-breaking): in all eigenstates $\mathcal{C}_{i j} \rightarrow 0$.

- Spin glass SG: in all eigenstates $\mathcal{C}_{i j} \neq 0$. The spectrum contains exponentially degenerate pairs of cat states which are superpositions of states with spin glass order and their Ising reversed counterparts. Equivalently, in the thermodynamic limit it consists entirely of states with broken Ising symmetry and spin glass long range order. Over each period, the order parameter returns to itself as detected by the depedence of $\mathcal{C}_{i j}$ within the period ${ }^{31}$.

- $\pi$-spin glass $\pi \mathrm{SG}$ : In all eigenstates $\mathcal{C}_{i j} \neq 0$. The spectrum contains pairs of cat states, with splitting is exponentially close to $\pi / T$. These are superpositions of states with spin glass order and their Ising reversed counterparts. Even in the thermodynamic limit these cannot be rearranged into states with explicitly broken Ising symmetry. Thus while the symmetry is broken as indicated by the two-point function, the catness is intrinsic. Over each period, the order parameter changes sign.

- $0 \pi$-paramagnet $0 \pi \mathrm{PM}$ : In all eigenstates $\mathcal{C}_{i j} \rightarrow 0$ in the bulk. However in open chains the spectrum comes in multiplets of four with splittings exponentially close to 0 and $\pi$; in closed chains the states are unique. Such phases are known as symmetryprotected topological phases, SPTs: trivial in the bulk, but with edge states on open chains. There is also interesting dynamics at the edge.

We emphasize that all these phases exhibit a breakdown of ETH in that the correlators fluctuate strongly between neighboring eigenstates. Thus, while an average over all states yields $T=\infty$ correlators, individual eigenstates do not, see Fig. 1. Also, the eigenstates exhibit area law entanglement which then also serves as an additional eigenstate diagnostic of the passage between any one of these phases and the ergodic phase. Interestingly, the two new phases can also be classified by means of local order parameters for time translation symmetry which is generated by $U(T)$ itself. Of these the $\pi \mathrm{SG}$ breaks time-translation symmetry in its bulk, while the $0 \pi \mathrm{PM}$ breaks it only at its boundaries: these provide examples of the "time crystals" first hypothesized for undriven systems although the term "spatio-temporally ordered" is perhaps more accurate. We describe the dynamical consequences of this identification below.

Finally, we note that the $\pi \mathrm{SG}$ is an exceptionally interesting phase. It is not merely stable to Ising invariant perturbations, instead it is absolutely stable ${ }^{32}$-i.e. it is stable to all weak perturbations that do not alter the drive period. ${ }^{45}$ The enlarged phase breaks an emergent Ising symmetry as well as time translation symmetry.

Late time states: Thus far we have made sharp statements about many body eigenstates. As these are in general not easy to prepare, it is important to ask what degree of universality is present in late time states reached by time evolution from more easily prepared initial states; and whether the above phases and transitions can be detected in such late time states. For the ergodic phase, but not for our case, ETH ensures that eigenstate and late time averages agree. Nevertheless, the late time states are sufficiently robust that the phase structure can indeed be detected. To see this, consider a general state

$$
|\chi(t)\rangle=\sum_{\alpha} c_{\alpha}\left|\psi_{\alpha}(t)\right\rangle=\sum_{\alpha} c_{\alpha} e^{-i \epsilon_{\alpha} t}\left|\phi_{\alpha}(t)\right\rangle
$$


which gives rise to the time dependent expectation value

$$
\langle\chi(t)|O| \chi(t)\rangle=\sum_{\alpha} \sum_{\beta} c_{\alpha}^{*} c_{\beta} e^{-i\left(\epsilon_{\alpha}-\epsilon_{\beta}\right) t}\left\langle\phi_{\beta}|O| \phi_{\alpha}(t)\right\rangle .
$$

For MBL-Floquet systems $\epsilon_{\alpha}-\epsilon_{\beta}$ is essentially continuously distributed in the thermodynamic limit, except for the spliitings internal to the spectral multiplets of the kind discussed above. Thus at late times the expectation value reduces to its value in the quasi-diagonal ensemble

$$
\begin{aligned}
\lim _{n \rightarrow \infty}\langle\chi(t+n T)| O & |\chi(t+n T)\rangle \sim \\
& \sum_{\alpha} \sum_{\beta(\alpha)} c_{\alpha}^{*} c_{\beta(\alpha)}\left\langle\phi_{\alpha}(t)|O| \phi_{\beta(\alpha)}(t)\right\rangle,
\end{aligned}
$$

so the late time density matrix is effectively,

$$
\rho \sim \sum_{\alpha} \sum_{\beta(\alpha)} c_{\alpha}^{*} c_{\beta(\alpha)}\left|\phi_{\alpha}(t)\right\rangle\left\langle\phi_{\beta(\alpha)}(t)\right|,
$$

with $\beta(\alpha)$ the member of the multiplet that contains $\alpha$. Thus at late times, roughly half the parameters present in the specification of the initial state (the phases) can no longer be recovered by local measurements.

For the phases of our model Ising drive the following table lists the characteristic behavior of late time states:

- PM: synchronized and paramagnetic. Expectation values strictly periodic with $T$ with those of Ising odd operators vanishing for all starting states.

- SG: synchronized and break Ising symmetry. For an initial state that breaks Ising symmetry, one point functions of Ising odd operators are nonzero while for Ising symmetric initial states we need to examine the two point functions at large distances.

- $0 \pi$ PM: synchronized and paramagnetic, except at the boundary, where they exhibit period doubling.

- $\pi \mathrm{SG}$ : break Ising symmetry with period doubling. For an initial state that breaks Ising symmetry, one point functions of Ising odd operators are nonzero while for Ising symmetric initial states we need to examine the two point functions at large distances. Stroboscopic snapshots look like Fig 2. In regions of the $\pi$ SG phase lacking a microscopic Ising symmetry, generic local operators will exhibit period doubling; this has been seen in an experiment. ${ }^{5}$

Finally we turn to free fermion systems, which turn out to behave differently. Of these, Anderson localized systems share much with their MBL cousins but they do not exhibit dephasing and so exhibit late time states with no particular periodicity. For free fermion Floquet systems without Anderson localization, stroboscopic evolution with $H_{F}$ is believed to lead to late time states which are well captured by a generalized Gibbs ensemble (GGE)

$$
\rho \sim e^{-\sum_{\alpha} \lambda_{\alpha} I_{\alpha}}
$$

With the non-trivial but periodic intra-period evolution included, this has been called the periodic Gibbs ensemble (PGE) or the Floquet-GGE. It is worth noting that the PGE density matrix leads to a volume law entanglement entropy that is less than the infinite temperature value, thus confirming a lack of heating. ${ }^{34}$ The moral of this part of the story is that much less information survives in the free fermion late time states than does in the diagonal ensembles that describe Floquet-MBL systems but more than survives for the Floquet-ETH case.

Recent developments and outlook: In a flurry of work, the program of identifying stable interacting Floquet phases has been pushed quite far already. ${ }^{11-15}$ This builds on an essentially complete classification analogous to that of topological insulators and superconductors for free fermion systems. ${ }^{35}$ The free fermion classification classifies single-particle unitaries and does not always lead to stable many body phases upon the addition of weak interactions as is the case for the analogous question for undriven free fermion systems. ${ }^{36}$ Among the examples which is stable is the anomalous Floquet Anderson insulator $^{37}$ which exhibits chiral edge modes without delocalized bulk states and is readily realized via a binary drive that appears to be experimentally feasible. The free fermion classification is, of course, relevant to experiments that probe few particle physics.

Cold atomic systems, combining long coherence times and tunability of geometry, disorder and interactions, provide an ideal platform for testing those ideas. An important development is the demonstration ${ }^{38,39}$ of (static) MBL in a disordered two-dimensional optical lattice, finding a transition into a regime at which memory of the initial state with an asymmetric boson occupancy became long-lived. Very recently, an analogous study ${ }^{4}$ was undertaken on a Floquet system with a (quasi-)disorder potential oscillating in time around a non-zero mean. Here, the memory indicative of MBL disappears as the driving frequency is lowered, in keeping with the abovementioned predictions. ${ }^{27,28}$ Finally, a first experiment claiming the observation of a discrete time crystal in the time domain has also appeared. ${ }^{5}$ An experimental tour de force, it involves a mesoscopic system, with the experimental verification of the full spatio-temporal order in the $\pi \mathrm{SG}$ remaining an outstanding challenge.

An important line of work that is highly relevant to experiments is on pre-thermal regimes for Floquet systems wherein they can exhibit plateaux characterized by equilibration with an effective $H_{F}$ over a long period before finally heating up to the ergodic steady state. ${ }^{40,41}$ In principle this makes it possible to observe non-trivial effective phases, such as time crystals, even in systems that are not localized. Excitingly, a very recent experiment sees such behavior in a three dimensional system of nitrogen vacancy centers, ${ }^{6}$ also in the time domain, although the precise connection to pre-thermalization theory not settled. There clearly remains much scope for further experimental studies of the increasingly rich and complex phenomena in many body Floquet systems. 
1 Immanuel Bloch, Jean Dalibard, Wilhelm Zwerger, Manybody physics with ultracold gases, Rev. Mod. Phys. 80, 885 (2008)

2 U. Schollwock, The density-matrix renormalization group in the age of matrix product states, Ann. Phys.326, 96 (2011)

3 Rahul Nandkishore, David A. Huse Many body localization and thermalization in quantum statistical mechanics, Annual Review of Condensed Matter Physics6, 15-38 (2015)

4 Pranjal Bordia, Henrik Lüschen, Ulrich Schneider, Michael Knap, Immanuel Bloch, Periodically Driving a Many-Body Localized Quantum System, arxiv:1607.07868, ()

5 J. Zhang, P. W. Hess, A. Kyprianidis, P. Becker, A. Lee, J. Smith, G. Pagano, I.-D. Potirniche, A. C. Potter, A. Vishwanath, N. Y. Yao, C. Monroe, Observation of a Discrete Time Crystal, arXiv:1609.08684, (2016)

${ }^{6}$ Soonwon Choi, Joonhee Choi, Renate Landig, Georg Kucsko, Hengyun Zhou, Junichi Isoya, Fedor Jelezko, Shinobu Onoda, Hitoshi Sumiya, Vedika Khemani, Curt von Keyserlingk, Norman Y. Yao, Eugene Demler, Mikhail D. Lukin, Observation of discrete time-crystalline order in a disordered dipolar many-body system, arxiv:1610.08057, (2016)

7 Milena Grifoni, Peter Hänggi, Driven quantum tunneling, Phys. Rep. 304, 229 (1998)

8 Marin Bukov, Luca D'Alessio, Anatoli Polkovnikov, Universal High-Frequency Behavior of Periodically Driven Systems: from Dynamical Stabilization to Floquet Engineering, Advances in Physics 64, 139-226 (2015)

9 Andrew C. Potter, Takahiro Morimoto, Ashvin Vishwanath Topological classification of interacting $1 D$ Floquet phases, arXiv:1602.05194, ()

10 Frederik Nathan, Mark S. Rudner, Topological singularities and the general classification of Floquet-Bloch systems Frederik Nathan, Mark S. Rudner, arXiv:1506.07647, ()

11 C. W. von Keyserlingk, S. L. Sondhi, Phase Structure of 1d Interacting Floquet Systems I: Abelian SPTs, arXiv:1602.02157, ()

12 C. W. von Keyserlingk, S. L. Sondhi, 1D Many-body localized Floquet systems II: Symmetry-Broken phases, arXiv:1602.06949, ()

13 Rahul Roy, Fenner Harper, Abelian Floquet SPT Phases in 1D, arXiv:1602.08089, ()

14 Rahul Roy, Fenner Harper Periodic Table for Floquet Topological Insulators, arXiv:1603.06944, ()

15 Dominic V. Else, Chetan Nayak Classification of topological phases in periodically driven interacting systems, arXiv:1602.04804, ()

16 Andre Eckardt, Christoph Weiss, Martin Holthaus, Superfluid-insulator transition in a periodically driven optical lattice, Phys. Rev. Lett. 95, 260404 (2005)

17 Takashi Oka, Hideo Aoki, Photovoltaic Hall effect in graphene, Phys. Rev. B 79, 081406 (2009)

18 Pedro Ponte, Anushya Chandran, Z. Papic, Dmitry A. Abanin, Periodically driven ergodic and many-body localized quantum systems, Annals of Physics 353, 196 (2015)

19 Dmitry Abanin, Wojciech De Roeck, Francois Huveneers, Exponentially slow heating in periodically driven manybody systems, Phys. Rev. Lett. 115, 256803 (2015)

20 Achilleas Lazarides, Arnab Das, Roderich Moessner, Equi- librium states of generic quantum systems subject to periodic driving, Phys. Rev. E 90, 012110 (2014)

21 Marcos Rigol, Vanja Dunjko, Maxim Olshanii, Thermalization and its mechanism for generic isolated quantum systems, Nature 452, 854 (2008)

22 Anushya Chandran, S. L. Sondhi, Interaction stabilized steady states in the driven $O(N)$ model, Phys. Rev. B 93, 174305 (2016)

23 Roberta Citro, Emanuele G. Dalla Torre, Luca D'Alessio, Anatoli Polkovnikov, Mehrtash Babadi, Takashi Oka, Eugene Demler, Dynamical Stability of a Many-body Kapitza Pendulum, Annals of Physics 360, 694-710 (2015)

24 T. Prosen, Time Evolution of a Quantum Many-Body System: Transition from Integrability to Ergodicity in the Thermodynamic Limit, Phys. Rev. Lett. 80, 1808 (1998)

25 Achilleas Lazarides, Arnab Das, Roderich Moessner, Periodic thermodynamics of isolated systems, Phys. Rev. Lett. 112, 150401 (2014)

26 Pedro Ponte, Z. Papic, Francois Huveneers, Dmitry A. Abanin, Many-body localization in periodically driven systems, Phys. Rev. Lett. 114, 140401 (2015)

27 Dmitry Abanin, Wojciech De Roeck, Francois Huveneers, A theory of many-body localization in periodically driven systems, arxiv:1412.4752, (2014)

28 Achilleas Lazarides, Arnab Das, Roderich Moessner, Fate of many-body localization under periodic driving, Phys. Rev. Lett. 115, 030402 (2015)

29 Marin Bukov, Markus Heyl, David A. Huse, Anatoli Polkovnikov, Heating and many-body resonances in a periodically driven two-band system, Phys. Rev. B 93, 155132 (2016)

30 David A. Huse, Rahul Nandkishore, Vadim Oganesyan, Arijeet Pal, S. L. Sondhi, Localization protected quantum order, Phys. Rev. B 88, 014206 (2013)

31 Vedika Khemani, Achilleas Lazarides, Roderich Moessner, S. L. Sondhi, On the phase structure of driven quantum systems, Phys. Rev. Lett. 116, 250401 (2016)

32 C.W. von Keyserlingk, Vedika Khemani, S. L. Sondhi $A b$ solute Stability and Spatiotemporal Long-Range Order in Floquet systems, arXiv:1605.00639, (2016)

33 Dominic V. Else, Bela Bauer, Chetan Nayak, Floquet Time Crystals, arXiv:1603.08001, (2016)

34 Arnab Sen, Sourav Nandy, K. Sengupta, Entanglement generation in periodically driven integrable systems: dynamical phase transitions and steady state, arXiv:1511.03668 , ()

35 Frederik Nathan, Mark S. Rudner, Topological singularities and the general classification of Floquet-Bloch systems, arXiv:1506.07647, ()

36 Xue-Yang Song, Andreas P. Schnyder, Interaction effects on the classification of crystalline topological insulators and superconductors, arXiv:1609.07469, ()

37 Paraj Titum, Erez Berg, Mark S. Rudner, Gil Refael, Netanel H. Lindner, The anomalous Floquet-Anderson insulator as a non-adiabatic quantized charge pump, Phys. Rev. X 6, 021013 (2016)

38 Pranjal Bordia, Henrik P. Lüschen, Sean S. Hodgman, Michael Schreiber, Immanuel Bloch, Ulrich Schneider, Coupling Identical 1D Many-Body Localized Systems, Phys. Rev. Lett. 116, 140401 (2016)

39 Jae-yoon Choi, Sebastian Hild, Johannes Zeiher, Peter 
Schauß, Antonio Rubio-Abadal, Tarik Yefsah, Vedika Khemani, David A. Huse, Immanuel Bloch, Christian Gross, Exploring the many-body localization transition in two dimensions, Science 352, 1547 (2016)

${ }^{40}$ Ionut-Dragos Potirniche, Andrew C. Potter, Monika Schleier-Smith, Ashvin Vishwanath, Norman Y. Yao, Floquet symmetry-protected topological phases in cold atomic systems, arXiv:1610.07611, ()

41 Simon A. Weidinger, Michael Knap, Floquet prethermalization and regimes of heating in a periodically driven, interacting quantum system, arXiv:1609.09089, ()

42 These are generalizations in that they reduce to the familiar ideas in the setting of ergodic, time dependent Hamiltonian systems.
43 This formulation is not quite crisp (but the conclusion nonethess correct): in a periodically driven system one needs to allow for periodic modulation of all quantities and so the proper replacement for time independent steady states is instead synchronization in which they all exhibit periodicity with the driving period.

44 For static/Floquet erogdic systems, this reduces to the conventional notion of order in the standard ensembles of statistical mechanics as nearby/all eigenstates by ETH all yield the same answer.

45 A previous paper ${ }^{33}$ found a stability axis protected by an anti-unitary Ising symmetry. 